\title{
AN ADAPTIVE OPTIMAL CONTROL STRATEGY FOR DYNAMIC STABILITY ENHANCEMENT OF AC/DC POWER SYSTEMS
}

\author{
N. Rostamkolai \\ Member \\ Virginia Polytechnic Institute and State University \\ Blacksburg, VA 24061 \\ A.G. Phadke \\ Fellow
}

\begin{abstract}
This paper presents an adaptive optimal control strategy for enhancement of the integrated $A C / D C$ power system performance. The system modeling is general and applicable to large scale power systems with an embedded HVDC link. The control algorithm incorporates the nonlinear power system dynamic equations. A novel feature of this development is the use of real-time system measurements as inputs to the optimal controller. The closed loop optimal control law consists of both model and measurement based terms. The effectiveness of the optimal control algorithm in damping the electromechanical oscillations is illustrated with the use of a nine-bus $A C / D C$ system.
\end{abstract}

\section{INTRODUCTION}

For a power system with an embedded HVDC link, the fast acting converter control offers a feature for hierarchical control of the system following a system disturbance. The dynamic stability and voltage excursions of a power system with an embedded HVDC link can be significantly influenced by simultaneous high speed control of synchronous generator exciters and HVDC converters. The variation of the continuously changing operating condition of a power system requires an adaptive control strategy to respond to contingencies.

The term adaptive control strategy, as referred to in this paper, is somewhat different from the usage in control literature and it implies to a control strategy which considers the changing operating condition of the power system.

In the literature, references [1]-[4] used a simplified power system representation and investigated various conventional control strategies in order to control different system parameters such as rotor angle movement, $\mathrm{AC}$ power transfer, or frequency. Generally selection of the best control strategy involved many simulation runs to obtain the best choice of the controller parameters for optimum system performance. The effect of fast converter set point change to vary the $\mathrm{DC}$ power and control the generator rotor swing for a two machine system connected by a parallel $\mathrm{AC} / \mathrm{DC}$ transmission system was investigated in [1]. Damping the power swings in a parallel AC and DC system for a generator connected to an infinite bus with use of linearized power equation was discussed in [2]. Experimental studies for improvement of the transient stability of $\mathrm{AC}$ system with the

87 SM 498-9 A paper recommended and approved by the IEEE Power System Engineering Committee of the IEEE Power Englneering Society for presentation at the IEEE/PES 1987 Summer Meeting, San Francisco, California, July $12-17,1987$. Manuscript submitted January 28, 1987; made avallable for printing

Apri1 21, 1987.

\author{
W.F. Long \\ Senior Member \\ University of Wisconsin \\ Madison, WI 53706
}

\author{
J.S. Thorp \\ Senior Member \\ Cornell University \\ Ithaca, NY 14853
}

use of equal area criterion for computation of the rate of change of the $\mathrm{DC}$ power in a perturbed parallel $\mathrm{AC} / \mathrm{DC}$ system and consequent control of the converters' ignition and extinction angles was carried out in [3]. The effect of the DC power changes and control of the frequency dip for two asynchronous systems connected by an HVDC link was documented in [4].

Improvement of the power system performance with application of the optimal control theory was investigated in $[5,6,7]$. In all cases a synchronous generator was connected to an infinite bus by parallel $\mathrm{AC} / \mathrm{DC}$ transmission lines. In [5] the Park representation of the generator and the inverse cosine control method with integral feedback for the HVDC link was used to formulate the system model. The linearized version of the system nonlinear equations was used for design of the conventional, optimal, and suboptimal controllers. In design of the optimal controller, linearized system equations were utilized in conjunction with a quadratic type of performance measure. In [6] an attempt was made to improve the transient stability by optimal switching of the converter set points, and a simple generator model wás considered. The maximum principle is applied to the nonlinear form of the system equations for computation of the optimal set point switching (bang-bang control). In [7] various optimal and suboptimal controllers, including the strategies used in [5] and [6] were compared for stabilization of a parallel $\mathrm{AC} / \mathrm{DC}$ power system.

The method of [5] in addition to using a simplified $\mathrm{AC} / \mathrm{DC}$ power system uses the linearized system equations for design of the optimal controller which is no longer optimal as the values of the system states change. The method of [6] uses the nonlinear form of the system equations which requires the solution of the two boundary value problem and it is mainly applicable to systems with small order and simple geometry. The papers referred to for stabilization of the AC power system make valuable contributions. However, in application of the optimal control theory to a realistic power system the techniques presented in these papers have limited use, since they consider a simplified power system representation, linearized power system equations, and locally measured parameters.

The contribution of this paper is the development of an adaptive optimal control strategy for a large scale AC/DC power system which uses the nonlinear form of system state equation and real-time system-wide measurements. The objective is to damp out the electromechanical oscillations in a power system by simultaneous optimal control of the synchronous generator exciters and the HVDC converters. However, the technique proposed in this paper can be extended for minimization of the voltage excursions at critical buses, although it is not explicitly studied here. The paper also formulates a power system state variable model applicable to large scale AC systems with an embedded two terminal HVDC transmission system. 
A real-time measurement technique for field implementation of the optimal controller is proposed which is discussed in more detail in a following section. However, in the work presented here the state measurements are obtained from a transient stability program which simulates the power system dynamics.

\section{CONTROL STRATEGY}

The optimal control strategy developed in this paper uses the nonlinear representation of the power system equations. The nonlinear equations are linearized by the Taylor series expansion which forms the linearized system model. This provides the information about the system at the base operating point. State feedback and input matrices obtained from the linearized equations are used for solution of the Riccati equation. Consequently, the Riccati equation is solved off-line and the solution is used for computation of the model based component of the optimal control law.

The dynamics of the system as it moves away from the base operating point is incorporated in a term referred to as the residual. This residual appears as an additional term in the control law, constituting an adaptive optimal control law. This additional term which is the solution of a vector differential equation with the residual as its forcing function, makes a proper contribution to the value of the optimal inputs when the excursions are significantly large during the transient period. The values of the states, the nonlinear equations of the power system, and information regarding the linearized system model are used to compute the value of the residual in real-time. A prediction algorithm is used to predict the future value of the residual, so that it can be treated as an explicit function of time.

For field implementation, in order to compute the value of residual and optimal inputs, a positive sequence phasor measurement technique is proposed [8]. This real-time measurement technique performs the system-wide synchronized measurements at remote locations in a power system and transmits the data to power system control center via fast communication links. The computation of the optimal inputs is based upon this measured data. The high speed and high accuracy feature of this real-time measurement technique makes it ideal for use with the proposed control algorithm.

\section{Control Algorithm}

The main contribution of this paper to the problem of nonlinear system control is introduced here. For the first time an optimal control algorithm is formulated which uses the nonlinear representation of the system state equation and yields an adaptive optimal control law. In the following derivation it is shown that the optimal control is a closed loop feedback type of control law.

The meaning of various symbols used in the derivation is explained at the end of the paper in the section entitled 'Nomenclature'. The nonlinear system is expressed by:

$$
\dot{X}(t)=F(X(t), U(t))
$$

then the Taylor series expansion results in,

$$
\dot{X}(t)=F\left(X_{d}(t), U_{d}(t)\right)+A\left[X(t)-X_{d}(t)\right]+B\left[U(t)-U_{d}(t)\right]
$$

where the $\mathrm{A}$ and $\mathrm{B}$ matrices are partials of the function $F$ of equation (1) with respect to $\mathrm{X}(\mathrm{t})$ and $\mathrm{U}(\mathrm{t})$ evaluated at $X_{d}(t)$ and $U_{d}(t)$, respectively. This however results in time variable $A$ and $B$ matrices. At this point we introduce the assumption that the $\mathrm{A}$ and $\mathrm{B}$ matrices are to be evaluated at $X\left(t_{0}\right)$ and $U\left(t_{0}\right)$. Although at first this assumption might seem to be too restrictive, it will be shown later that the error caused by this assumption is captured in the residual term of the state equation. Defining the translations:

$$
\begin{aligned}
& Z(t)=X(t)-X_{d}(t) \\
& V(t)=U(t)-U_{d}(t)
\end{aligned}
$$

and substitution of equations (3) and (4) into equation (2) leads to:

$$
\dot{X}(t)=F\left(X_{d}(t), U_{d}(t)\right)+A Z(t)+B V(t)
$$

Expressing the original form of the nonlinear state equation with inclusion of the terms from the Taylor series expansion results in:

$$
\begin{aligned}
\dot{X}(t)= & F\left(X_{d}(t), U_{d}(t)\right)+A Z(t)+B V(t)+ \\
& {\left[F(X(t), U(t))-F\left(X_{d}(t), U_{d}(t)\right)-A Z(t)-B V(t)\right] }
\end{aligned}
$$

Now a term $\dot{X}_{d}(t)$ is subtracted from both sides of the equation (6), and the equation is expressed as:

$$
\dot{Z}(t)=A Z(t)+B V(t)+f(t)
$$

where,

$$
f(t)=F(X(t), U(t))-A Z(t)-B V(t)-\dot{X}_{d}(t)
$$

$f(t)$ is the residual mentioned earlier, and as expressed by equation (8) captures the inaccuracies resulting from the evaluation of the $A$ and $B$ matrices at initial value of the states and inputs rather than their desired values. The $f(t)$ is computed during the control interval from the measured value of the state variables and its future value is extrapolated by the predictor. As more state measurements become available and time progresses, the accuracy in prediction of the future values of the residual improves.

To minimize the error in the states and inputs of the original system from the desired values, the following regulator type of performance measure is utilized for the translated system of equation (7).

$$
J(V)=\frac{1}{2} \int_{t_{0}}^{t}\left[Z^{T}(t) Q Z(t)+V^{T}(t) R V(t)\right] d t
$$

The optimal control law for the translated system is [15],

$$
V^{*}(t)=R^{-1} B^{T}[g(t)-K(t) Z(t)]
$$

where $\mathrm{K}(\mathrm{t})$ is the solution of the Riccati equation:

$$
\begin{gathered}
\dot{K}(t)=-K(t) A(t)-A^{T}(t) K(t)+K(t) B(t) R^{-1}(t) B^{T}(t) K(t)-Q(t) \\
K\left(t_{f}\right)=0
\end{gathered}
$$

The term $\mathrm{g}(\mathrm{t})$ is the solution of equation:

$$
\dot{g}(t)=-\left[A^{T}-K(t) B R^{-1} B^{T} \lg (t)+K(t) f(t), \quad g\left(t_{f}\right)=0\right.
$$

The pre-disturbance state measurement vector initializes the $f(t)$ vector. As soon as the new state measurements are obtained they are used to compute and store $f(t)$. The collection of measurements and computation of $f(t)$ during a fault are avoided, since they are grossly in error. Immediately after the removal of a fault, as the new measurements arrive they are used to compute $f(t)$ and the prediction algorithm is executed to reevaluate the future values of $f(t)$ from their previously stored values. For this purpose a moving average predictor with a window of four samples is used. Then the vector differential equation (12) is solved for $g(t)$ with the new estimate of $f(t)$, thus forming an adaptive optimal control law. It is important to notice that according to the presented control algorithm, the solution of the Riccati equation is independent of the residual $f(t)$. Since the system $A$ and $B$ matrices remain unchanged, the time variable $K(t)$ matrix remains unchanged. Therefore, the Riccati equation is solved off-line and its solution is stored. 


\section{Real-Time Measurement Technique}

Many measurement techniques are found in the literature which measure the magnitude and angle of complex voltages of one phase. These measurement techniques are susceptible to errors resulting from unbalanced conditions and harmonic components of the waveform. Hence, these methods produce inaccurate values of the measured parameters. The technique described in [8] measures digitally filtered positive sequence voltage and current phasors, which reduces the inaccuracies to a minimal value, in an appropriate time frame for real-time monitoring and control applications.

This technique was developed as a result of the introduction of microprocessors into substations for power system protection. These devices-better known as computer relaysmeasure the positive sequence voltage and current phasors at system buses in real-time. Synchronized sampling clocks are required in various substations in order to have the measurements in a common frame of reference. For clocks synchronized to within about 10 microseconds, the accuracy of the measured phase angles are within 0.2 degrees of their correct value on a $60 \mathrm{~Hz}$ basis.

The use of this technique for the presented control algorithm provides the generator phase voltage, line current, and frequency. Further, the generator $\mathrm{q}$-axis complex voltage can be computed and the value of the angle of this voltage (in polar coordinates) represents the value of generator rotor angle. Also, the generator speed can be deduced from the frequency measurement. Therefore, all the necessary information in regard to the value of parameters for the adaptive optimal controller is made available.

\section{POWER SYSTEM MODELING}

In order to test the control strategy presented here, realtime measurements are necessary. An AC/DC transient stability program is developed to provide the values of the states during the transient period, and simulate the performance of the system with or without the optimal controller. The effects of turbines and governors are excluded, since the time constant associated with the change in mechanical power is 4 to 5 times larger than the control interval chosen for this study. Therefore, it is assumed that the turbine shaft power $P_{m}$ will remain constant during the control interval.

The stability program requires usual types of component models, and the selected models are described next. In addition, a state variable model (different from the component models) is required for computation of $A, B$, and $K(t)$ matrices. They are described in a later section. The component models are used in the transient stability program which uses the Newton-Raphson technique for solution of the network equations, and a fixed time step Runge-Kutta method for numerical integration of the differential equations. The sequential technique of [9] was used for implementation of the HVDC model. The sequential technique represents the DC link by real and reactive loads which are updated in each iteration of Newton-Raphson method as the converter AC bus voltages change after each iteration.

\section{Component Models}

The models of different components of a power system as selected for this study are discussed here. The selection of simple models for transient stability program is to reduce the computational complexity of the program. 'These component models are connected through a complex transmission network. The necessary equations for modeling of each dynamic element of the power system as used in the transient stability program are described in the following.

\section{Generator Model:}

Proper generator modeling is an important factor for investigation of power system dynamics. A number of well accepted synchronous machine models are available which use the Park frame of reference $[9,10]$. In addition to a number of differential equations required for the generator model, two additional differential equations represent the mechanical equations of motion:

$$
\begin{gathered}
\frac{2 H}{\omega_{0}} \dot{\omega}+D\left(\omega-\omega_{0}\right)=P_{m}-P_{e} \\
\dot{\delta}=\omega-\omega_{0}
\end{gathered}
$$

The optimal control of the power system of interest is a dynamic stability consideration, and as documented in [11] a third order generator model satisfies the requirements for this study. Hence, the following equation is sufficient to describe the generator d-axis dynamics.

$$
\dot{E}_{q}^{\prime}=\frac{1}{T_{d 0}^{*}}\left(E_{f d}-E_{l}\right)
$$

\section{Exciter Model:}

A wide variéty of standard exciter models are available for control of the generator field voltage. The IEEE Type 1 exciter model with an auxiliary input as shown in [12] is adapted for this study. The time domain differential equation representation of the exciter model with this auxiliary input is as follows.

$$
\begin{gathered}
\dot{V}_{y}=\frac{1}{T_{r}}\left(E_{t}-V_{y}\right) \\
\dot{V}_{r}=\frac{1}{T_{a}}\left\lfloor-V_{r}+\left(-V_{y}-V_{z}+V_{r e f}+V_{s}\right) K_{a}\right] \\
\dot{V}_{2}=\frac{1}{T_{f}}\left\{\frac{K_{f}}{T_{e}}\left[V_{r}-\left(S_{e}+K_{e}\right) E_{f d}\right]-V_{z}\right\} \\
\dot{E}_{f d}=\frac{1}{T_{e}}\left[V_{r}-\left(S_{e}+K_{e}\right) E_{f d}\right]
\end{gathered}
$$

This is a fourth order exciter model. Thus, a generator and its excitation system forms a system of order seven. The auxiliary voltage $V_{s}$ is the exciter control input.

\section{HVDC Model:}

The quasi-steady state model of the HVDC converter is used for representation of the DC link [13]. This model expresses the DC voltage as a function of the primary converter AC voltage, converter transformer tap ratio, valve ignition angle, transformer commutating reactance, and the DC current. The DC voltages at rectifier and inverter in per unit are obtained from the following equations. The conventional base values from reference [14] are adapted for expression of the equations in per unit.

$$
\begin{gathered}
V_{d r}^{p u}=a_{r} E_{r}^{p u} \cos \alpha-\frac{\pi}{6} X_{c r}^{p u} I_{d c}^{p u} \\
V_{d i}^{p u}=a_{i} E_{l}^{p u} \cos \gamma-\frac{\pi}{6} X_{c l}^{p u} I_{d c}^{p u} \\
V_{d r}^{p u}=R_{d}^{p u} I_{d c}^{p u}+V_{d l}^{p u}
\end{gathered}
$$

The relationship between the two terminal stations is expressed by equation (22) as an algebraic equation. Even though this is not an exact relationship due to the inductance of smoothing reactors and the DC line, the associated time constant is small and it can be neglected for dynamic stabil- 
ity studies. The time constant of the control circuitry is also neglected for the same reason.

The above equations are necessary to compute the real and reactive powers of the $\mathrm{DC}$ link at the converter station. Neglecting the converter losses,

$$
P_{a c}^{p u}=P_{d r}^{p u}=V_{d r}^{p u} I_{d c}^{p u}
$$

where $P_{d r}$ is the per unit DC power at rectifier. The per unit reactive power at rectifier for support of the DC link is computed from:

$$
Q_{d r}^{p u}=a_{r} k_{r} E_{r}^{p u} I_{d c}^{p u} \sin \varphi
$$

where $\varphi$ is the power factor angle and according to reference [9] it is computed from:

$$
\cos \varphi=\frac{1}{2 k_{r}}[\cos \alpha+\cos (\alpha+u)]
$$

And,

$$
k_{r}=\frac{\sqrt{[\cos 2 \alpha-\cos 2(\alpha+u)]^{2}+[2 u+\sin 2 \alpha-\sin 2(\alpha+u)]^{2}}}{4[\cos \alpha-\cos (\alpha+u)]} \text { (26) }
$$

For an inverter $\alpha$ is replaced by $\gamma$ and the subscript $r$ is replaced by $\mathrm{i}$ in equations (23) through (26). Therefore, the DC real and reactive powers are highly dependent on the converter ignition and extinction angles.

\section{State Variable Model}

In this section the power system state variable model and modification of the component equations are described. This model is necessary in order to calculate the matrices A, B, and $K(t)$ of equations (7) and (11). The network is represented by its admittance matrix. Instead of the usual load and HVDC terminal representation by specified real and reactive powers in the stability program, in this system model the loads and the HVDC terminals are represented by the base value admittances in the admittance matrix. This does not impose a limitation on the loads and HVDC terminals which in reality are not constant impedances, since the departures from constant impedances are reflected in the state measurements obtained from the actual system and the residual computation. The dimension of the network admittance matrix is equal to the number of $\mathrm{AC}$ buses. To form the connection of the generators to the network, their q-axis synchronous reactances are used to form additional buses. Hence, the dimension of the network admittance matrix is increased by the number of generators in the system. The network reduction technique reduces the size of the admittance matrix, by preserving the identity of the generator $\mathrm{q}$-axis buses in addition to the reference bus and eliminating all the other system buses. Therefore, the partitioned admittance matrix is:

$$
\left[\begin{array}{l}
I_{1} \\
I_{2}
\end{array}\right]=\left[\begin{array}{ll}
Y_{1} & Y_{2} \\
Y_{3} & Y_{4}
\end{array}\right]\left[\begin{array}{l}
E_{1} \\
E_{2}
\end{array}\right]
$$

where $I_{1}$ is the vector of current injections at non-generating buses, $I_{2}$ is the vector of current injections at generating buses, $E_{1}$ is the vector of voltages at non-generating buses, $E_{2}$ is the vector of the $\mathrm{q}$-axis voltages at generating buses. The Kron formula is used to obtain the reduced order admittance matrix, or

$$
Y_{e q}=Y_{4}-Y_{3} Y_{1}^{-1} Y_{2}
$$

This equivalent admittance matrix represents the reduced order static model of the network.

Next the generator dynamic equations are modified in order to include interaction of the generators through the network, and to create the desirable state variable form of the equations. The complex power of generator $i$ is:

$$
S_{i}=E_{q i} I_{t i}{ }^{*} \quad, \quad i=1, \ldots, j
$$

where $\mathrm{j}$ is the number of generators in the system, $E_{q i}$ is the fictitious bus voltage behind the $\mathrm{q}$-axis synchronous reactance, and $I_{t i}$ is the generator i terminal current. Then, the complex power in terms of the admittances of the admittance matrix of equation (28) is:

$$
S_{i=} E_{q i}\left[\sum_{k=1}^{j+1} Y_{i k} E_{k}\right]^{*}, \quad i=1, \ldots j
$$

The generator $i$ real and reactive powers are:

$$
\begin{array}{ll}
P_{l}=\operatorname{Re}\left[S_{i}\right] \quad, \quad i=1, \ldots j \\
Q_{i}=\operatorname{Im}\left[S_{i}\right] \quad, \quad i=1, \ldots j
\end{array}
$$

Substitution of the $P_{i}$ relation of equation (31) into the equation (13) for each generator forms the primitive state variable form of the swing equation which will be modified later. Equation (14) remains unchanged, and the third dynamic equation for a cylindrical rotor generator is:

$$
\dot{E}_{q}^{\prime}=\frac{1}{T_{d 0}}\left[E_{f d}-E_{q}\right]
$$

As shown in [15] the differential equation dependency on $E_{q}^{\prime}$ is eliminated and a new differential equation in terms $E_{q}$ replaces equation (33). It is also shown that the magnitude of the generator d-axis current is:

$$
I_{d i}=\frac{Q_{i}}{E_{q i}}, \quad i=1, \ldots . j
$$

which is used to express the generator terminal voltage (not a state variable) in terms of the q-axis voltages and the rotor angles (state variables) for connection of the generator and exciter equations.

It is important to notice that the network model completely ignores the effect of the DC power change. To include this factor, the network sensitivity technique is used to modify the swing equation (13). This method shows the approximate change in the output power of each generator as a result of a change in DC power [16]. The rectifier and inverter powers are related by the following equation [15].

$$
P_{d r}=\frac{V_{d i}+R_{d} \frac{P_{d i}}{V_{d i}}}{V_{d i}} P_{d i}
$$

Thus, equation (35) shows the relationship between the rectifier and inverter powers as a function of inverter DC voltage and DC line resistance. Now the rate of change of the DC power at rectifier in terms of the change in inverter power is:

$$
\Delta P_{d r}=\left[1+\frac{2 R_{d}}{V_{d i}^{2}} P_{d i}^{0}\right] \Delta P_{d i}
$$

From the network sensitivity method (generation shift factor) a change in rectifier power causes a change in the generator i power by the following expression:

$$
G_{l, r}=\frac{\Delta P_{i}}{\Delta P_{d r}}, \quad G_{i, r}=-\frac{X_{r, t i}}{x_{q i}}
$$

Also, a change in the inverter power causes a change in generator i power by:

$$
G_{i, i}=\frac{\Delta P_{i}}{\Delta P_{d i}}, \quad G_{i, i}=-\frac{X_{i, t i}}{x_{q i}}
$$

The inverter power is a positive generation while the rectifier power is a negative generation. In order to maintain consistency, the sign of the generation shift factor corresponding 
to the change in rectifier power is reversed and the rectifier power increment is assumed to be a positive quantity. Since the network sensitivity technique uses the linearized equations of the system, the superposition theorem applies and the total change in the generator $i$ power as a result of a change in $\mathrm{DC}$ link power transfer is:

$$
\Delta P_{l}=-G_{l,} \Delta P_{d r}+G_{l, l} \Delta P_{d l}
$$

where $\Delta P_{d r}$ and $\Delta P_{d i}$ are both positive quantities in the above equation. Now substitution of equation (36) into equation (39) results in:

$$
\Delta P_{i}=-G_{l, d} 1+\frac{2 R_{d}}{V_{d l}^{2}} P_{d i}^{0} \mid \Delta P_{d l}+G_{l, j} \Delta P_{d l}
$$

or the total change in generator $i$ power due to a change in HVDC link power is:

$$
\Delta P_{l}=\Omega_{l} \Delta P_{d l}
$$

where,

$$
\Omega_{i}=-G_{l, r l} 1+\frac{2 R_{d}}{V_{d l}^{2}} P_{d i l}^{0}+G_{l, l}
$$

Finally the swing equation of (13) is put in the proper state variable form as follows:

$$
\frac{2 H}{\omega_{0}} \dot{\omega}+D\left(\omega-\omega_{0}\right)=P_{m}-R e[S]-\Omega \Delta P_{d}
$$

which includes the effect of DC power modulation.

The state variable model of the large scale system requires modification of the generator equations for representation of the overall power system. The reason for the modifications is to formulate the proper state variable form of the equations which expresses the dynamics of an interconnected system. The state and input vectors of the nonlinear state equation (1) are defined as follows:

$$
\begin{gathered}
X(t)=\left[\begin{array}{llllll}
X_{G 1} & X_{G 2} & X_{G 3} & \ldots & X_{G j}
\end{array}\right]^{T} \\
U(t)=\left[\begin{array}{llllll}
U_{E 1} & U_{E 2} & U_{E 3} & \ldots & U_{E j} & U_{d d}
\end{array}\right]^{T}
\end{gathered}
$$

$X_{G 1}$ through $X_{G j}$ are the state vectors for generators 1 through j. $U_{E 1}$ through $U_{E j}$ are the input vectors for excitation systems 1 through $\mathrm{j}$, and $U_{d c}$ is the input for the HVDC link. The definition of each component of the state vector is:

$$
X_{G}=\left[\begin{array}{lllllll}
\omega & \delta & E_{q} & V_{y} & V_{z} & V_{r} & E_{f d}
\end{array}\right]^{T}
$$

where $V_{y}, V_{z}$, and $V_{r}$ are the exciter internal voltages. And the definition of each component of the input vector is:

$$
\begin{gathered}
U_{E}=\left[V_{\text {ref }}+V_{s}\right] \\
U_{d c}=\left[\Delta P_{d l}\right]
\end{gathered}
$$

\section{CONTROLLER IMPLEMENTATION}

The schematic representation of the optimal controller is shown in Figure 1. The synchronized real-time measurements are processed to compute and dispatch the optimal inputs to the system exciters and rectifiers. This optimal controller is formulated in the transient stability program for computation of the optimal inputs. For a selected control interval of 5 seconds, the adaptive optimal controller block executes the following steps in every 5 cycles of the simulation time. First, it collects and stores the measurements in an array. Second, it computes $f(t)$. Third, it estimates the future values of residual from the previous values, using a moving average predictor with a window of 4 samples. Fourth, it computes the new value of $g(t)$ from the new knowledge of $f(t)$, and also computes $Z(t)$. Fifth, if necessary it reads the new value of $K(t)$ from off-line computations, and computes the optimal inputs. Sixth, it maintains the computed value of the optimal inputs for the next 5 cycles and dispatchs them to the control locations.

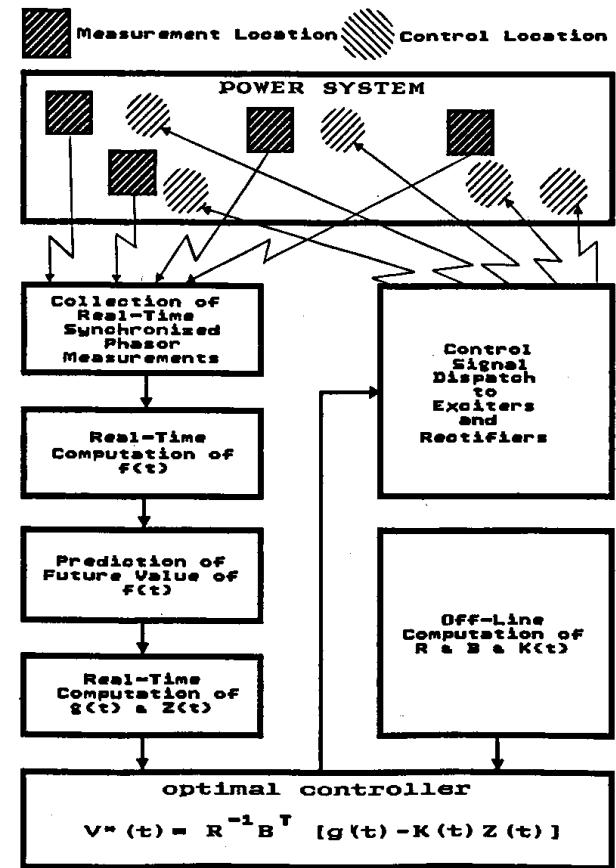

Figure 1. Representation of the optimal controller

\section{SIMULATION RESULTS}

The one line diagram of the nine-bus system is shown in Figure 2. The system has two generators, three transformers with two of them having the capability of off-nominal turns ratio, a two terminal HVDC link, a load bus, and an infinite bus. The disturbance simulated is a three phase fault on one of the parallel AC lines between buses 2 and 3, and the fault location is close to bus 3 . The three phase fault is modeled as a low impedance connection between bus 3 and ground. The duration of the fault simulated is three cycles. Finally, one of the $\mathrm{AC}$ lines is removed to clear the fault.

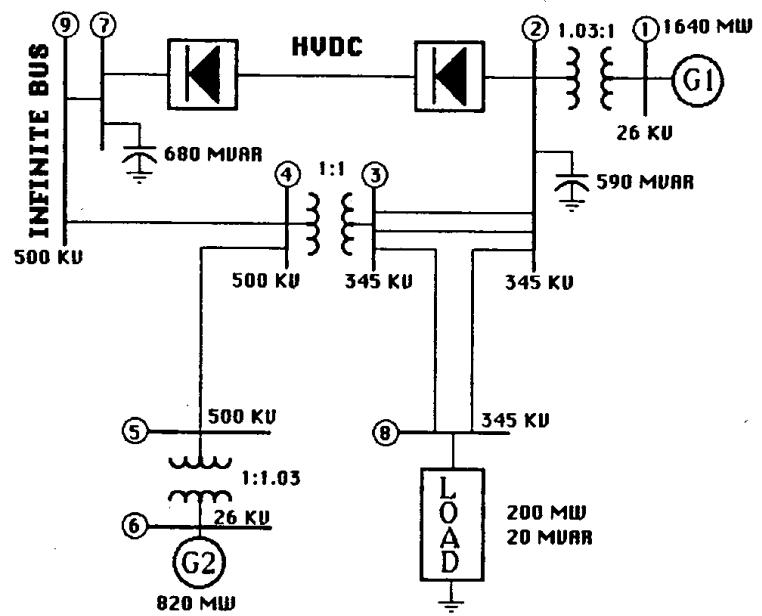

Figure 2. Integrated AC/DC system 
The simulation results of this system are illustrated and discussed in the following. The rotor angles of generator 1 and 2 (DELTA) are plotted in degrees. Also, the exciter optimal inputs (VS) and the DC current are plotted in per unit. The time axis is in seconds. The plotted parameters for the optimally controlled system are compared to the parameters of the system with classical control strategy. In this paper classical control strategy refers to the control strategy which maintains a constant DC current at the rectifier and a constant DC voltage at the inverter. It does not refer to any control strategy involving the modulation of $\mathrm{AC}$ or $\mathrm{DC}$ controls with the use of some feedback signals.

Figure 3 shows the simulation results when the time variable solution of the Riccati equation is used. The future values of the vector $f(t)$ are predicted by a straight line, obtained from computation of the moving average of the previous values of $f(t)$ from the state measurements. The rotor angle swings of generators 1 and 2 have a very small and negligible final value error. The error in the final value of the optimal inputs are however zero.
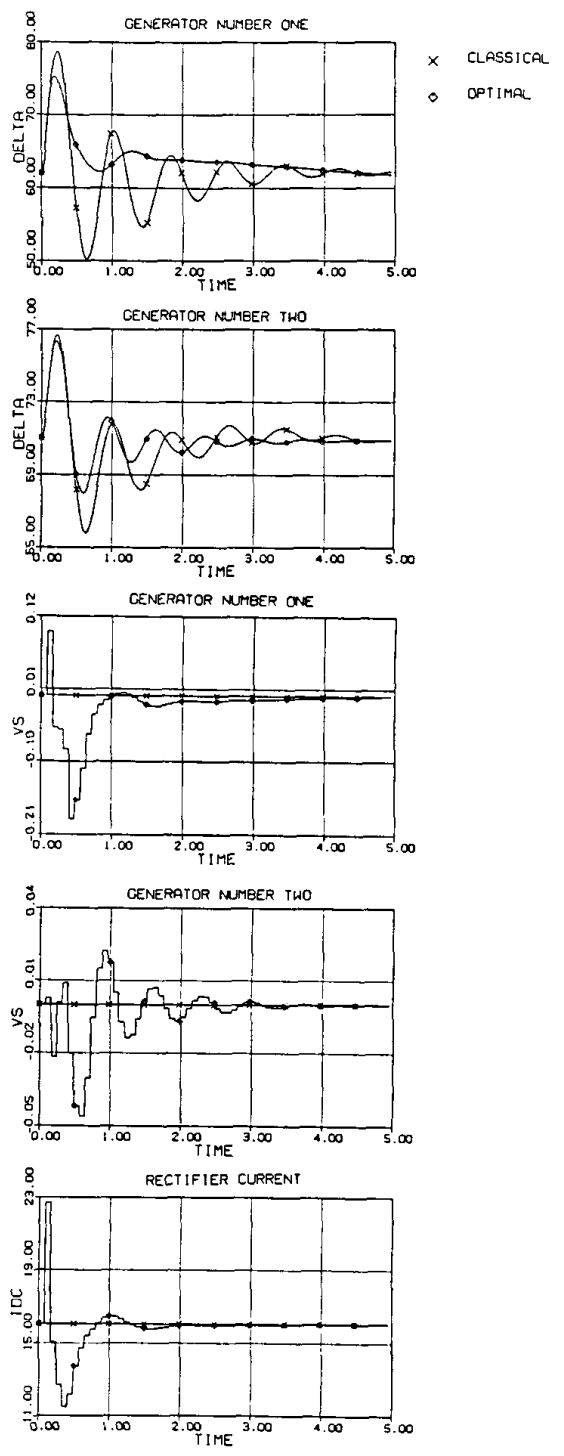

Figure 3. Simulation results with exact optimal control law
The simulation results using the steady state solution of the Riccati equation are not shown, since their trajectories are almost identical to the trajectories of Figure 3 . The only disadvantage of using the steady state solution of the Riccati equation instead of the time variable solution of this equation is the small errors in the final value of the optimal inputs. However, use of the steady state solution of the Riccati equation reduces computational burden and memory requirements significantly.

In obtaining the value of optimal inputs from the optimal control law, the term $\mathrm{g}(\mathrm{t})$ which is the solution of equation (12) is needed. However, if the steady state solution of $g(t)$ is used rather than the actual value of $g(t)$ from the differential equation (12), the need for time consuming iterative solution of this differential equation is eliminated and the term $g(t)$ is computed simply from a matrix multiplication as follows.

$$
g(t)=\left[A^{T}-K B R^{-1} B^{T}\right]^{-1} K f
$$

The steady state solution of the Riccati equation must be used for this approximation. The use of the steady state solution of $\mathrm{g}(\mathrm{t})$ and also the steady state solution of the Riccati equation result in an approximate optimal control law. Figure 4 shows the simulation results for the approximate optimal control law. The rotor angle swings of generators 1 and 2 as shown in the figure illustrate no substantial difference between the exact and approximate approaches.
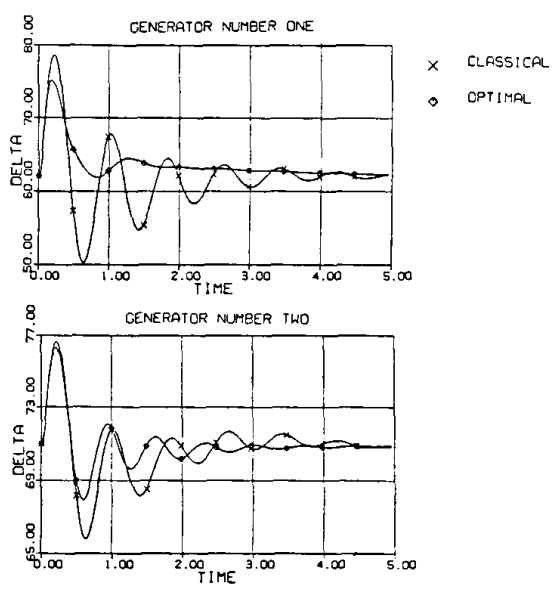

Figure 4. Simulation results with approximate optimal control law

As shown in Figures 3 and 4, the optimal controller performs very well and improves the power system performance significantly. The rotor angle swings of generator 1 are damped out very rapidly, but in case of generator 2 it takes a longer time. This is due to the fact that controllability is governed by the system structure. Even though the control objective for this study was damping the generator rotor angle swings, a performance criterion can be formulated to minimize the voltage excursions at critical load buses with or without consideration for damping the system electromechanical oscillations.

\section{CONCLUSIONS}

Simulation results illustrated the excellent performance of the optimal controller in damping the electromechanical oscillations. Close similarities are seen in the trajectory of the rotor angle swings when the steady state and time variable 
solution of the Riccati equation is used. Comparison of the rotor angle swings of generators 1 and 2 , when the exact and approximate optimal control law is used shows that there are no significant differences in the two trajectories. Therefore, the approximate control law can be implemented rather than the exact optimal control law for the adaptive optimal control of the system. The approximate optimal control law is computationally much faster than the exact optimal control law. This is due to the fact that the numerical integration for solution of the differential equation involving the term $g(t)$ as required by the exact optimal control law is replaced by a simple matrix multiplication for the approximate optimal control law. Also, the need for storage of the time variable solution of the Riccati equation is eliminated. Consequently, implementation of the approximate control law removes the computational time limitations which can be a serious problem for implementation of the exact optimal control law as an on-line optimal controller.

\section{ACKNOWLEDGEMENT}

This work was sponsored by the ASEA Power Systems Center - Wisconsin contract number 808649-1.

\section{NOMENCLATURE}

$X(t)$ : state vector

$X(t)$ : desired value of the state vector

$U(t)$ : input vector

$U(t)$ : desired value of the input vector

$\omega$ : generator speed

$\omega_{0}:$ generator reference speed

$\delta$ : generator rotor angle

$\mathrm{H}$ : inertia constant

D : damping coefficient

$P_{m} \quad$ : generator mechanical power

$P_{e}^{m} \quad$ : generator electrical power

$E_{t} \quad$ : terminal voltage

$E_{g} \quad$ : voltage behind q-axis synchronous reactance

$E^{q}:$ voltage proportional to field flux linkages

$E_{l}{ }^{q}$ : voltage proportional to field current

$E_{f d}$ : field voltage acting along the q-axis

$I_{t}$ : terminal current

$I_{d} \quad:$ d-axis component of the terminal current

$T_{\infty}:$ d-axis open circuit time constant

$V_{y}$ : output voltage of the filter block

$V_{z} \quad$ : output voltage of the feedback block

$V_{r} \quad$ : output voltage of the regulator block

$V_{\text {ref }}$ : exciter reference voltage

$V_{s}:$ auxiliary input for control purposes

$V_{t} \quad:$ rectifier DC voltage

$V_{d i}:$ inverter DC voltage

$I_{d c}:$ DC current

$E_{r} \quad$ : primary AC voltage at rectifier

$E_{i} \quad$ : primary AC voltage at inverter

$a_{r}:$ transformer tap ratio at rectifier

$a_{i} \quad:$ transformer tap ratio at inverter

$X_{r} \quad:$ transformer short circuit reactance at rectifier

$X_{c i}:$ transformer short circuit reactance at inverter

$R_{d}:$ DC line resistance

$\alpha \quad$ : rectifier ignition angle

$\gamma \quad$ : inverter extinction angle

$u$ : overlap angle

$P_{i} \quad$ : electrical power of generator $i$

$P_{d r}:$ rectifier DC power

$P_{d i}$ : inverter DC power

$G_{i, r}:$ generator i shift factor for a change in rectifier power

$G_{i, j}$ : generator i shift factor for a change in inverter power

$x_{q i}: \mathrm{q}$-axis synchronous reactance of generator $\mathrm{i}$

$\vec{X}_{r, i i}, X_{i, t i}$ : corresponding components of reactance matrix

\section{REFERENCES}

1. H.A. Peterson, D.K. Reitan, and A.G. Phadke, "Parallel Operation of AC and DC Power Transmission," IEEE Transactions on Power Apparatus and Systems, Vol. PAS-84, No. 1, pp. 15-19, January 1965.

2. H.A. Peterson, and P.C. Krause, "Damping of Power Swings in a Parallel AC and DC system," IEEE Transactions on Power Apparatus and Systems, Vol. PAS-85, No. 12, pp. 1231-1239, December 1966.

3. T. Machida, "Improving Transient Stability of AC System by Joint Usage of DC Systems," IEEE Transactions on Power Apparatus and Systems, Vol. PAS-85, No. 3, pp. 226-232, March 1966.

4. Y. Yoshida, T. Machida, and N. Hingorani, "Ana$\log$ Computer Study of Automatic Frequency Ratio Control on an HVDC Transmission System," IEEE Transactions on Power Apparatus and Systems, Vol. PAS-87, No. 3, pp. 796-807, March 1968.

5. P.K. Dash, B. Puthal, O.P. Malik, and G.S. Hope. "Transient Stability and Optimal Control of Parallel AC-DC Power Systems," IEEE Transactions on Power Apparatus and Systems, Vol. PAS-95, No. 3, pp. 811-820, May/June 1976.

6. H. Kobayashi, and K. Ichiyanagi, “ Improvement of the Transient Stability by Optimal Switching Control of Parallel AC-DC Power System," IEEE Transactions on Power Apparatus and Systems, Vol. PAS-97, No. 4, pp. 1140-1148, July/August 1978.

7. A.H.M.A. Rahim, I.M. El-Amin, "Stabilization of a High Voltage AC-DC Power System, I. Evaluation of Control Strategies," IEEE Power Engineering Society Winter Meeting, New York, N.Y., Paper No. 85 WM 068-2, 1985.

8. A.G. Phadke, J.S. Thorp, and M.G. Adamiak, “ A New Measurement Technique for Tracking Voltage Phasors, Local System Frequency, and Rate of Change of Frequency," IEEE Transactions on Power Apparatus and Systems, Vol. PAS-102, No. 5, pp. 1025-1033, May 1983.

9. J. Arrillaga, C.P. Arnold, and B.J. Harker, Computer Modelling of Electric Power Systems, John Wiley and Sons, Inc., 1983.

10. G.W. Stagg, and A.H. El-Abiad, Computer Methods in Power System Analysis, McGraw-Hill, Inc., 1968.

11. V.A. Venikov, V.A. Stroev, and Mohsen A.H. Tawfik, " Optimal Control of Transients in Electrical Power Systems Containing Controlled Reactors, Part 2: Optimal Control Problem Solution," IEEE Transactions on Power Apparatus and Systems, Vol. PAS-100, No. 9, pp. 4271-4280, September 1981 .

12. P.M. Anderson, and A.A. Fouad, Power System Control and Stability, The Jowa State University Press, 1977.

13. E.W. Kimbark, Direct Current Transmission, John Wiley and Sons, Inc., 1971.

14. A.G. Phadke, and J.H. Harlow, "Unbalanced Converter Operation," IEEE Transactions on Power Apparatus and Systems, Vol. PAS-85, No. 3, pp. 233-239, March 1966.

15. N. Rostamkolai, Adaptive Optimal Control of $A C / D C$ Systems, Virginia Polytechnic Institute and State University, Ph.D. Dissertation (Available at Virginia Polytechnic Institute and State University Library), 1986.

16. A.J. Wood, and B.F. Wollenberg, Power Generation, Operation, and Control, John Wiley and Sons, Inc., 1984. 